\title{
Evaluation of the Prevalence of Childhood Autism Awareness amongst Medical Professionals in Pakistan
}

\author{
NAWAL NOOR ${ }^{1}$, MUHAMMAD TALHA ${ }^{1}$, SAED AFTAB AHMAD ${ }^{2}$, MAHNOOR MOHYDIN ${ }^{3}$, SYED SAQIB ALI SHAH ${ }^{3}$, \\ SHAHMEER MOHYDIN ${ }^{4}$, FILZAH FAHEEM ${ }^{3}$ \\ ${ }^{1}$ Medical Student, Lahore Medical and Dental College (LMDC), Lahore \\ ${ }^{2}$ Professor of Paediatrics, Rashid Latif Medical College, Hameed Latif Hospital, Lahore. \\ ${ }^{3}$ House Officer, Services Hospital, Lahore. \\ ${ }^{4}$ Medical Student, Shalamar Medical and Dental College (SMDC), Lahore \\ Correspondence to Mahnoor Mohydin, E-mail: mahnoormohydin@gmail.com , Telephone\# +923066246667
}

\begin{abstract}
Aim: To evaluate the level of awareness regarding childhood autism among medical professionals of Pakistan. Methodology: A cross-sectional descriptive survey was conducted among 105 medical students and health-care professionals to estimate their knowledge regarding Autism in Pakistan. By random sampling method, questionnaires were distributed during the month of April 2020. IRB approval was obtained.

Results: The results have highlighted that medical students have significant knowledge of Autism Spectrum Disorder while Post Graduate trainees and House Officers have moderate knowledge. However, it is alarming to see that there is lack of knowledge among Medical Officers and Consultants. Due to this deficient knowledge gap, medical practitioners are unaware of the severity of this disorder in the region. The results emphasize that there is either initial lack of medical school curriculum or over time knowledge of medical professionals decreases due to which they are unable to identify the prevalent disorders.

Conclusion: ASD is a pertinent regional health concern. Lack of clinical knowledge of this disorder amongst healthcare professionals, needs to be addressed by application of awareness systems. This will improve diagnosis and redesign treatment modalities alongside strategize preventive measures.

Keywords: Autism Spectrum Disorder, Medical, Student, Child Development Disorders
\end{abstract}

\section{INTRODUCTION}

The introduction of Autism as a disorder dates back to 1943, when child psychologist, Leo Kanner observed 11 children who were behaving differently than others. The deduction made was that the children had an inherent problem, which restricted them from regular social contacts $^{1}$. Autism Spectrum Disorder (ASD) is a clinically complex neurodevelopmental disorder that primarily displays in social communication deficits and repetitive motor behaviours. Although different studies have revealed that affected individuals differ greatly from each other, but impairment in communication plus social reciprocity is common in all ${ }^{2}$. Most of the time, the children exhibit having delayed speech; they are not interested in their peers, avoid eye contact, show limited response to their name, and have little shared attention with parents. Autistic children also exhibit repetitive behaviours like stacking objects, lining, or flapping as well as paying special attention to spinning objects. All these symptoms appear before three years of age and usually have male predominance. Clinical diagnosis is necessary for confirming the disease because there is a lack of reliable biomarkers ${ }^{3}$.

There are various aetiologies of this disorder ranging from genetic and environmental factors to acquired infections, pregnancy complications, pollution and mental health issues. However, due to underreporting of its prevalence, etiological factors and symptomology, there is limited treatment in developing countries like Pakistan.

Received on 13-02-2021

Accepted on 15-05-2021
Pakistan is a developing country in Southeast Asia, with a population of more than 197 million, which is the fifth most populous nation in the world ${ }^{4}$. More than $38 \%$ of this population is under the age of 15 years ${ }^{5}$. Although significant improvements have been undertaken in the last few decades in improving the provision of healthcare services, areas of mental health and learning disability still remains largely neglected. Hence, in the broadspectrum of evaluation many psychological issues prevail either being unaddressed or having limited capacity. Only about $3 \%$ of the country's annual budget is spent on health ${ }^{5}$. Mental health, including child mental health, has no separate budget allocation. Psychiatric services in Pakistan are curbed to psychiatry departments in teaching hospitals or privately run clinics ${ }^{6}$. Only around 340 psychiatrists and a handful of child psychiatrists are responsible for providing the much-needed services ${ }^{6}$.

According to the Autism Society of Pakistan, there are more than 350,000 children who are suffering from this disease. The field experts further confirm that the autism disorder is increasing over time. It is necessary to bring attention of physicians to this specific disease because it is not related to physical injury or laboratory testing. Thus, it cannot be easily diagnosed and requires clinical analysis ${ }^{7}$.

It has been observed that professionals have various misconceptions about the salient features of the disease, which results in mismanagement of the patient as treatment plans are not put properly in practice ${ }^{8}$. Recent studies have emphasized that in developing countries, the prevalence rate is $0.9 / 1000$, and most of the affected children are from rural areas ${ }^{9}$. There is poor prognosis and the health improvement is slow in nature, 
which impacts the well-being of children subject to the disorder. Therefore, there is an urgent need for responsible authorities to measure the knowledge gaps; which is causing a major inability for proper provision of medical care as the status-quo remains unknown. Moreover, a short-term plan with remedial actions should also be implemented on immediate basis alongside planning for long-term strategies ${ }^{9-10}$.

This specific study was planned to evaluate childhood autism awareness among the medical professionals of Pakistan in order to evaluate the prevalence of lack of awareness regarding ASD so that a proper intervention plan can be developed accordingly.

\section{METHODOLOGY}

This cross-sectional descriptive study was conducted by random sampling method among 105 medical professionals in Pakistan during April 2020 for one month. The medical professionals included house officers, medical students, Post graduate trainees, and medical officers. IRB approval was obtained and all the participants consented to be a part of the study. Data was collected through an online survey by using a selfadministered questionnaire, accessing knowledge regarding Autism. The survey consisted of questions regarding "Autism Awareness." Questions were prepared using the knowledge related to Childhood Autism among Medical Professionals (KCAMP) as well as other studies. "Autism Awareness" was accessed with the help of a Likert scale in which five different options were provided to the participants, such as SD (Strongly Disagree), D (Disagree), N (Neutral), A (Agree) and SA (Strongly Agree). Strongly disagree means they lack knowledge regarding the specific question while strongly agree directly translates to having high knowledge. Data was analysed using SPSS 23 . The primary association among the categorical variables was evaluated with the help of the chi-square test, and the value $\mathrm{P}<0.05$ was statistically significant.

\section{RESULTS}

By analysing the opinions of respondents at different educational levels, it can be said that there is an unsatisfactory knowledge of autism in healthcare benefactors which are unswervingly associated to diagnostics so if they failed to reach a diagnosis in the first place how can they treat the disorder.

The consent form was sent to 150 people from which $70 \%$ agreed to give consent to take part in the study. Of the 105 participants, there were more females than males. Age varied between 22 to 40 years of age, with most medical students being 22 years of age, while other participants were above the age of 22 years. The majority were medical students and the other $17.1 \%$ accumulatively included Postgraduate trainees, Houseofficers, Medical Officers and Consultants. A significant portion, $41 \%$ claimed that they had never been around someone who was suffering from Autism (Table 1).

There is evident lack of awareness regarding this disorder, with $39 \%$ being likely aware of autism, and $20 \%$ being moderately aware. There was no significant difference in the level of awareness between genders. Of the ones aware, $28 \%$ were female and $33 \%$ male. There were more medical students that were up to date and agreed that autism is a rare condition in Pakistan in comparison to one Post Graduate trainee being in disagreement with this statement. Among all the respondents, most medical students agreed that genetics plays a crucial role in the aetiology of autism. While, altogether more house-officers and medical students agreed that exposure to heavy metals and other toxins enhances the risk of autism development. Regarding whether the nature of the disorder is neurodevelopmental and if low birth weight can be a cause, 38 medical students, 5 house officers, and 3 medical officers agreed. However, 4 medical students and 1 house officer disagreed with the statement that children with autism start showing symptoms by the age of $8-10$ months. Further, $46 \%$ medical students were aware that children with autism face communication problems, and they show symptoms like delayed speech. Mostly, it was also agreed that it is sometimes difficult to differentiate that either the child has autism or ADHD. 30 medical students and 4 house officers agreed that although autistic children lack the proper communication but they can appropriately use their body and hand gestures. Regarding, whether autistic children face difficulty in exhibiting emotional cues such as affection (as well as they do not have any perception of fear and anger), 35 medical students, 6 house officers, and 2 PG trainees agreed.

Medical students, medical officers and house officers altogether $70.50 \%$ agreed that doctors use the child's behavioural and developmental history to evaluate them to make a diagnosis. While, $13.30 \%$ strongly agreed.

Table 1: Demographic Analysis

\begin{tabular}{|c|c|c|}
\hline Characteristics & Categories & Frequency\% \\
\hline \multirow{3}{*}{ Gender } & Female & $57(54.3)$ \\
\hline & Male & $48(45.7)$ \\
\hline & Total & $105(100.0)$ \\
\hline \multirow[t]{6}{*}{ Educational Status } & Consultant & $1(1.0)$ \\
\hline & House officer & $10(9.5)$ \\
\hline & Medical officer & $5(4.8)$ \\
\hline & Medical student & $87(82.9)$ \\
\hline & PG trainee & $2(1.9)$ \\
\hline & Total & $105(100.0)$ \\
\hline \multirow{4}{*}{$\begin{array}{l}\text { Do you know or have } \\
\text { you spent time around } \\
\text { someone with Autism } \\
\text { (ASD)? }\end{array}$} & Maybe & $21(20.0)$ \\
\hline & No & $43(41.0)$ \\
\hline & Yes & $41(39.0)$ \\
\hline & Total & $105(100.0)$ \\
\hline
\end{tabular}

Among all, 52.90\% medical students exhibited sound knowledge of autism, and they rejected the idea that autism can be diagnosed with the help of medical tests such as blood tests. Medical students and house officers agreed that behavioural and speech therapies, along with communication therapy, are the main-stay treatment for autistic children. They also agreed that with the proper treatment, most of the children outgrow autism. Medical students, house officers and medical officers by $62.90 \%$ agreed that parents of autistic children face severe stress, which increases with the age of child 
because they require extra attention for their medical condition (Table 2).

Table 2: Autism Awareness According to Education Level

\begin{tabular}{|c|c|c|c|c|c|c|c|}
\hline & & Consultant & $\begin{array}{l}\text { House } \\
\text { Officer }\end{array}$ & $\begin{array}{l}\text { Medical } \\
\text { Officer }\end{array}$ & $\begin{array}{l}\text { Medical } \\
\text { student }\end{array}$ & $\begin{array}{l}\text { PG } \\
\text { trainee }\end{array}$ & Total \\
\hline \multirow{4}{*}{$\begin{array}{l}\text { Autism is a rare condition in } \\
\text { Pakistan as compared to the } \\
\text { world. }\end{array}$} & Agree & $0(0.00 \%)$ & $3(30.00 \%)$ & $3(60.00 \%)$ & $26(29.90 \%)$ & $0(0.00 \%)$ & $32(30.50 \%)$ \\
\hline & Disagree & $0(0.00 \%)$ & $1(10.00 \%)$ & $2(40.00 \%)$ & $19(21.80 \%)$ & $1(50.00 \%)$ & $23(21.90 \%)$ \\
\hline & Neutral & $1(100.00 \%)$ & $6(60.00 \%)$ & $0(0.00 \%)$ & $33(37.90 \%)$ & $1(50.00 \%)$ & $41(39.00 \%)$ \\
\hline & Strongly disagree & $0(0.00 \%)$ & $0(0.00 \%)$ & $0(0.00 \%)$ & $3(3.40 \%)$ & $0(0.00 \%)$ & $3(2.90 \%)$ \\
\hline \multirow{4}{*}{$\begin{array}{l}\text { Total } \\
\text { Genetics play major role in the } \\
\text { etiology of autism. }\end{array}$} & & $1(100.00 \%)$ & $10(1000 \%)$ & $5(100.00 \%)$ & $87(100.00 \%)$ & $2(100.00 \%)$ & $105(100 \%)$ \\
\hline & Agree & $0(0.00 \%)$ & $9(90.00 \%)$ & $2(40.00 \%)$ & $39(44.80 \%)$ & $1(50.00 \%)$ & $51(48.60 \%)$ \\
\hline & Disagree & $0(0.00 \%)$ & $0(0.00 \%)$ & $1(20.00 \%)$ & $9(10.30 \%)$ & $1(50.00 \%)$ & $11(10.50 \%)$ \\
\hline & Strongly disagree & $0(0.00 \%)$ & $0(0.00 \%)$ & $0(0.00 \%)$ & $6(6.90 \%)$ & $0(0.00 \%)$ & $6(5.70 \%)$ \\
\hline \multirow{6}{*}{$\begin{array}{l}\text { Total } \\
\text { Exposure to heavy metals and } \\
\text { toxins has been suggested by } \\
\text { evidence to increase the risk of } \\
\text { developing autism. }\end{array}$} & & $1(100.00 \%)$ & $10(100 \%)$ & $5(100.00 \%)$ & $87(100.00 \%)$ & $2(100.00 \%)$ & $105(100)$. \\
\hline & Agree & $1(100.00 \%)$ & $4(40.00 \%)$ & $2(40.00 \%)$ & $37(42.50 \%)$ & $1(50.00 \%)$ & $45(42.90 \%)$ \\
\hline & Disagree & $0(0.00 \%)$ & $1(10.00 \%)$ & $2(40.00 \%)$ & $10(11.50 \%)$ & $1(50.00 \%)$ & $14(13.30 \%)$ \\
\hline & Neutral & $0(0.00 \%)$ & $5(50.00 \%)$ & $0(0.00 \%)$ & $33(37.90 \%)$ & $0(0.00 \%)$ & $38(36.20 \%)$ \\
\hline & Strongly agree & $0(0.00 \%)$ & $0(0.00 \%)$ & $0(0.00 \%)$ & $6(6.90 \%)$ & $0(0.00 \%)$ & $6(5.70 \%)$ \\
\hline & Strongly disagree & $0(0.00 \%)$ & $0(0.00 \%)$ & $1(20.00 \%)$ & $1(1.10 \%)$ & $0(0.00 \%)$ & $2(1.90 \%)$ \\
\hline $\begin{array}{l}\text { Total } \\
\text { Autism is a neurodevelopmental } \\
\text { disorder and can be associated } \\
\text { with low birth weight. }\end{array}$ & Strongly disagree & $0(0.00 \%)$ & $0(0.00 \%)$ & $0(0.00 \%)$ & $1(1.10 \%)$ & $0(0.00 \%)$ & $1(1.00 \%)$ \\
\hline \multirow{6}{*}{$\begin{array}{l}\text { Total } \\
\text { Children with autism can show } \\
\text { symptoms by the age of } 8-10 \\
\text { months. }\end{array}$} & & $1(100.00 \%)$ & $10(100.00 \%)$ & $5(100.00 \%)$ & $87(100.00 \%)$ & $2(100.00 \%)$ & $105(100 \%)$ \\
\hline & Agree & $0(0.00 \%)$ & $6(60.00 \%)$ & $4(80.00 \%)$ & $38(43.70 \%)$ & $1(50.00 \%)$ & $49(46.70 \%)$ \\
\hline & Disagree & $0(0.00 \%)$ & $0(0.00 \%)$ & $1(20.00 \%)$ & $21(24.10 \%)$ & $0(0.00 \%)$ & $22(21.00 \%)$ \\
\hline & Neutral & $1(100.00 \%)$ & $3(30.00 \%)$ & $0(0.00 \%)$ & $19(21.80 \%)$ & $1(50.00 \%)$ & $24(22.90 \%)$ \\
\hline & Strongly Agree & $0(0.00 \%)$ & $0(0.00 \%)$ & $0(0.00 \%)$ & $5(5.70 \%)$ & $0(0.00 \%)$ & $5(4.80 \%)$ \\
\hline & Strongly disagree & $0(0.00 \%)$ & $1(10.00 \%)$ & $0(0.00 \%)$ & $4(4.60 \%)$ & $0(0.00 \%)$ & $5(4.80 \%)$ \\
\hline & $1(100.00 \%)$ & $10(100.00 \%)$ & $5(100.00 \%)$ & $87(100.00 \%)$ & $2(100.00 \%)$ & $105(100 \%)$ \\
\hline \multirow{4}{*}{$\begin{array}{l}\text { Children with autism may have a hard } \\
\text { time interacting with others and have } \\
\text { problem with communication like } \\
\text { delayed speech. }\end{array}$} & Agree & $1(100.00 \%)$ & $6(60.00 \%)$ & $3(60.00 \%)$ & $40(46.00 \%)$ & $1(50.00 \%)$ & $51(48.60 \%)$ \\
\hline & Disagree & $0(0.00 \%)$ & $0(0.00 \%)$ & $0(0.00 \%)$ & $1(1.10 \%)$ & $0(0.00 \%)$ & $1(1.00 \%)$ \\
\hline & Neutral & $0(0.00 \%)$ & $1(10.00 \%)$ & $0(0.00 \%)$ & $3(3.40 \%)$ & $0(0.00 \%)$ & $4(3.80 \%)$ \\
\hline & Strongly agree & $0(0.00 \%)$ & $3(30.00 \%)$ & $2(40.00 \%)$ & $43(49.40 \%)$ & $1(50.00 \%)$ & $49(46.70 \%)$ \\
\hline & $1(100.00 \%)$ & $10(100.00 \%)$ & $5(100.00 \%)$ & $87(100.00 \%)$ & $2(100.00 \%)$ & $105(100 \%)$ \\
\hline \multirow{2}{*}{$\begin{array}{l}\text { It is sometimes difficult to } \\
\text { differentiate between a child with } \\
\text { autism and ADHD. }\end{array}$} & Agree & $1(100.00 \%)$ & $8(80.00 \%)$ & $3(60.00 \%)$ & $42(48.30 \%)$ & $1(50.00 \%)$ & $55(52.40 \%)$ \\
\hline & Disagree & $0(0.00 \%)$ & $0(0.00 \%)$ & $0(0.00 \%)$ & $7(8.00 \%)$ & $0(0.00 \%)$ & $7(6.70 \%)$ \\
\hline Autistic child usually does not & Agree & $0(0.00 \%)$ & $6(60.00 \%)$ & $2(40.00 \%)$ & $35(40.20 \%)$ & $2(100.00 \%)$ & $45(42.90 \%)$ \\
\hline respond to emotional cues ; such & Disagree & $0(0.00 \%)$ & $1(10.00 \%)$ & $1(20.00 \%)$ & $22(25.30 \%)$ & $0(0.00 \%)$ & $24(22.90 \%)$ \\
\hline as affection and has no & Neutral & $1(100.00 \%)$ & $1(10.00 \%)$ & $1(20.00 \%)$ & $18(20.70 \%)$ & $0(0.00 \%)$ & $21(20.00 \%)$ \\
\hline perception of fear and anger. & Strongly Agree & $0(0.00 \%)$ & $0(0.00 \%)$ & $1(20.00 \%)$ & $8(9.20 \%)$ & $0(0.00 \%)$ & $9(8.60 \%)$ \\
\hline & Strongly disagree & $0(0.00 \%)$ & $2(20.00 \%)$ & $0(0.00 \%)$ & $4(4.60 \%)$ & $0(0.00 \%)$ & $6(5.70 \%)$ \\
\hline Total & & $1(100.00 \%)$ & $10(100.00 \%)$ & $5(100.00 \%)$ & $87(100.00 \%)$ & $2(100.00 \%)$ & $105(100 \%)$ \\
\hline Adults with autism may follow a & Agree & $0(0.00 \%)$ & $9(90.00 \%)$ & $5(100.00 \%)$ & $54(62.10 \%)$ & $2(100.00 \%)$ & $70(66.70 \%)$ \\
\hline rigid routine every day and get & Disagree & $0(0.00 \%)$ & $0(0.00 \%)$ & $0(0.00 \%)$ & $6(6.90 \%)$ & $0(0.00 \%)$ & $6(5.70 \%)$ \\
\hline anxious if it changes. & Neutral & $1(100.00 \%)$ & $1(10.00 \%)$ & $0(0.00 \%)$ & $12(13.80 \%)$ & $0(0.00 \%)$ & $14(13.30 \%)$ \\
\hline & Strongly Agree & $0(0.00 \%)$ & $0(0.00 \%)$ & $0(0.00 \%)$ & $15(17.20 \%)$ & $0(0.00 \%)$ & $15(14.30 \%)$ \\
\hline Total & & $1(100.00 \%)$ & $10(100.00 \%)$ & $5(100.00 \%)$ & $87(100.00 \%)$ & $2(100.00 \%)$ & $105(100 \%)$ \\
\hline Autistic people can notice small & Agree & $1(100.00 \%)$ & $7(70.00 \%)$ & $2(40.00 \%)$ & $40(46.00 \%)$ & $1(50.00 \%)$ & $51(48.60 \%)$ \\
\hline details, patterns, smells and & Disagree & $0(0.00 \%)$ & $0(0.00 \%)$ & $1(20.00 \%)$ & $3(3.40 \%)$ & $0(0.00 \%)$ & $4(3.80 \%)$ \\
\hline sounds that others do not. & Neutral & $0(0.00 \%)$ & $3(30.00 \%)$ & $0(0.00 \%)$ & $25(28.70 \%)$ & $0(0.00 \%)$ & $28(26.70 \%)$ \\
\hline & Strongly Agree & $0(0.00 \%)$ & $0(0.00 \%)$ & $2(40.00 \%)$ & $18(20.70 \%)$ & $1(50.00 \%)$ & $21(20.00 \%)$ \\
\hline & Strongly disagree & $0(0.00 \%)$ & $0(0.00 \%)$ & $0(0.00 \%)$ & $1(1.10 \%)$ & $0(0.00 \%)$ & $1(1.00 \%)$ \\
\hline Total & & $1(100.00 \%)$ & $10(100.00 \%)$ & $5(100.00 \%)$ & $87(100.00 \%)$ & $2(100.00 \%)$ & $105(100 \%)$ \\
\hline It is possible for adults with & Agree & $0(0.00 \%)$ & $6(60.00 \%)$ & $3(60.00 \%)$ & $45(51.70 \%)$ & $0(0.00 \%)$ & $54(51.40 \%)$ \\
\hline autism to go undiagnosed. & Disagree & $0(0.00 \%)$ & $2(20.00 \%)$ & $1(20.00 \%)$ & $20(23.00 \%)$ & $1(50.00 \%)$ & $24(22.90 \%)$ \\
\hline & Neutral & $1(100.00 \%)$ & $1(10.00 \%)$ & $0(0.00 \%)$ & $15(17.20 \%)$ & $1(50.00 \%)$ & $18(17.10 \%)$ \\
\hline & Strongly Agree & $0(0.00 \%)$ & $1(10.00 \%)$ & $0(0.00 \%)$ & $2(2.30 \%)$ & $0(0.00 \%)$ & $3(2.90 \%)$ \\
\hline & Strongly disagree & $0(0.00 \%)$ & $0(0.00 \%)$ & $1(20.00 \%)$ & $5(5.70 \%)$ & $0(0.00 \%)$ & $6(5.70 \%)$ \\
\hline Total & & $1(100.00 \%)$ & $10(100.00 \%)$ & $5(100.00 \%)$ & $87(100.00 \%)$ & $2(100.00 \%)$ & $105(100 \%)$ \\
\hline
\end{tabular}




\begin{tabular}{|c|c|c|c|c|c|c|c|}
\hline \multirow{5}{*}{$\begin{array}{l}\text { Doctors evaluate the child's } \\
\text { developmental and behavioral } \\
\text { history to help make the } \\
\text { diagnosis. }\end{array}$} & Agree & $1(100.00 \%)$ & $6(60.00 \%)$ & $4(80.00 \%)$ & $61(70.10 \%)$ & $2(100.00 \%)$ & $74(70.50 \%)$ \\
\hline & Disagree & $0(0.00 \%)$ & $1(10.00 \%)$ & $1(20.00 \%)$ & $2(2.30 \%)$ & $0(0.00 \%)$ & $4(3.80 \%)$ \\
\hline & Neutral & $0(0.00 \%)$ & $1(10.00 \%)$ & $0(0.00 \%)$ & $11(12.60 \%)$ & $0(0.00 \%)$ & $12(11.40 \%)$ \\
\hline & Strongly Agree & $0(0.00 \%)$ & $1(10.00 \%)$ & $0(0.00 \%)$ & $13(14.90 \%)$ & $0(0.00 \%)$ & $14(13.30 \%)$ \\
\hline & Strongly disagree & $0(0.00 \%)$ & $1(10.00 \%)$ & $0(0.00 \%)$ & $0(0.00 \%)$ & $0(0.00 \%)$ & $1(1.00 \%)$ \\
\hline \multicolumn{2}{|l|}{ Total } & $1(100.00 \%)$ & $10(100.00 \%)$ & $5(100.00 \%)$ & $87(100.00 \%)$ & $2(100.00 \%)$ & $105(100 \%)$ \\
\hline \multirow{5}{*}{$\begin{array}{l}\text { Autism can be detected by } \\
\text { medical testing; such as blood } \\
\text { tests. }\end{array}$} & Agree & $0(0.00 \%)$ & $2(20.00 \%)$ & $1(20.00 \%)$ & $4(4.60 \%)$ & $0(0.00 \%)$ & $7(6.70 \%)$ \\
\hline & Disagree & $1(100.00 \%)$ & $1(10.00 \%)$ & $2(40.00 \%)$ & $46(52.90 \%)$ & $1(50.00 \%)$ & $51(48.60 \%)$ \\
\hline & Neutral & $0(0.00 \%)$ & $4(40.00 \%)$ & $1(20.00 \%)$ & $22(25.30 \%)$ & $1(50.00 \%)$ & $28(26.70 \%)$ \\
\hline & Strongly agree & $0(0.00 \%)$ & $0(0.00 \%)$ & $0(0.00 \%)$ & $2(2.30 \%)$ & $0(0.00 \%)$ & $2(1.90 \%)$ \\
\hline & Strongly disagree & $0(0.00 \%)$ & $3(30.00 \%)$ & $1(20.00 \%)$ & $13(14.90 \%)$ & $0(0.00 \%)$ & $17(16.20 \%)$ \\
\hline \multicolumn{2}{|l|}{ Total } & $1(100.00 \%)$ & $10(100.00 \%)$ & $5(100.00 \%)$ & $87(100.00 \%)$ & $2(100.00 \%)$ & $105(100 \%)$ \\
\hline \multirow{5}{*}{$\begin{array}{l}\text { Dietary intervention such as } \\
\text { removing certain food from diet or } \\
\text { using vitamins and mineral } \\
\text { supplements is one of treatment } \\
\text { options. }\end{array}$} & Agree & $0(0.00 \%)$ & $5(50.00 \%)$ & $0(0.00 \%)$ & $19(21.80 \%)$ & $1(50.00 \%)$ & $25(23.80 \%)$ \\
\hline & Disagree & $1(100.00 \%)$ & $2(20.00 \%)$ & $3(60.00 \%)$ & $26(29.90 \%)$ & $1(50.00 \%)$ & $33(31.40 \%)$ \\
\hline & Neutral & $0(0.00 \%)$ & $3(30.00 \%)$ & $2(40.00 \%)$ & $34(39.10 \%)$ & $0(0.00 \%)$ & $39(37.10 \%)$ \\
\hline & Strongly Agree & $0(0.00 \%)$ & $0(0.00 \%)$ & $0(0.00 \%)$ & $4(4.60 \%)$ & $0(0.00 \%)$ & $4(3.80 \%)$ \\
\hline & Strongly disagree & $0(0.00 \%)$ & $0(0.00 \%)$ & $0(0.00 \%)$ & $4(4.60 \%)$ & $0(0.00 \%)$ & $4(3.80 \%)$ \\
\hline \multicolumn{2}{|l|}{ Total } & $1(100.00 \%)$ & $10(100.00 \%)$ & $5(100.00 \%)$ & $87(100.00 \%)$ & $2(100.00 \%)$ & $105(100 \%)$ \\
\hline \multirow{5}{*}{$\begin{array}{l}\text { Positive reinforcement and } \\
\text { parental counseling on training } \\
\text { techniques can go a long way } \\
\text { with children with autism. }\end{array}$} & Agree & $1(100.00 \%)$ & $7(70.00 \%)$ & $2(40.00 \%)$ & $40(46.00 \%)$ & $1(50.00 \%)$ & $51(48.60 \%)$ \\
\hline & Disagree & $0(0.00 \%)$ & $1(10.00 \%)$ & $1(20.00 \%)$ & $1(1.10 \%)$ & $0(0.00 \%)$ & $3(2.90 \%)$ \\
\hline & Neutral & $0(0.00 \%)$ & $1(10.00 \%)$ & $0(0.00 \%)$ & $10(11.50 \%)$ & $0(0.00 \%)$ & $11(10.50 \%)$ \\
\hline & Strongly agree & $0(0.00 \%)$ & $1(10.00 \%)$ & $2(40.00 \%)$ & $35(40.20 \%)$ & $1(50.00 \%)$ & $39(37.10 \%)$ \\
\hline & Strongly disagree & $0(0.00 \%)$ & $0(0.00 \%)$ & $0(0.00 \%)$ & $1(1.10 \%)$ & $0(0.00 \%)$ & $1(1.00 \%)$ \\
\hline \multicolumn{2}{|l|}{ Total } & $1(100.00 \%)$ & $10(100.00 \%)$ & $5(100.00 \%)$ & $87(100.00 \%)$ & $2(100.00 \%)$ & $105(100 \%)$ \\
\hline \multirow{4}{*}{$\begin{array}{l}\text { Behavior therapy, speech therapy } \\
\text { and communication therapy are } \\
\text { the mainstay treatments for } \\
\text { autism. }\end{array}$} & Agree & $1(100.00 \%)$ & $6(60.00 \%)$ & $1(20.00 \%)$ & $39(44.80 \%)$ & $2(100.00 \%)$ & $49(46.70 \%)$ \\
\hline & Disagree & $0(0.00 \%)$ & $0(0.00 \%)$ & $0(0.00 \%)$ & $1(1.10 \%)$ & $0(0.00 \%)$ & $1(1.00 \%)$ \\
\hline & Neutral & $0(0.00 \%)$ & $2(20.00 \%)$ & $1(20.00 \%)$ & $5(5.70 \%)$ & $0(0.00 \%)$ & $8(7.60 \%)$ \\
\hline & Strongly agree & $0(0.00 \%)$ & $2(20.00 \%)$ & $3(60.00 \%)$ & $42(48.30 \%)$ & $0(0.00 \%)$ & $47(44.80 \%)$ \\
\hline \multicolumn{2}{|l|}{ Total } & $1(100.00 \%)$ & $10(100.00 \%)$ & $5(100.00 \%)$ & $87(100.00 \%)$ & $2(100.00 \%)$ & $105(100 \%)$ \\
\hline \multirow{5}{*}{$\begin{array}{l}\text { With proper treatment, most } \\
\text { children with autism eventually } \\
\text { outgrow autism. }\end{array}$} & Agree & $1(100.00 \%)$ & $5(50.00 \%)$ & $3(60.00 \%)$ & $41(47.10 \%)$ & $2(100.00 \%)$ & $52(49.50 \%)$ \\
\hline & Disagree & $0(0.00 \%)$ & $2(20.00 \%)$ & $2(40.00 \%)$ & $9(10.30 \%)$ & $0(0.00 \%)$ & $13(12.40 \%)$ \\
\hline & Neutral & $0(0.00 \%)$ & $2(20.00 \%)$ & $0(0.00 \%)$ & $23(26.40 \%)$ & $0(0.00 \%)$ & $25(23.80 \%)$ \\
\hline & Strongly agree & $0(0.00 \%)$ & $0(0.00 \%)$ & $0(0.00 \%)$ & $12(13.80 \%)$ & $0(0.00 \%)$ & $12(11.40 \%)$ \\
\hline & Strongly disagree & $0(0.00 \%)$ & $1(10.00 \%)$ & $0(0.00 \%)$ & $2(2.30 \%)$ & $0(0.00 \%)$ & $3(2.90 \%)$ \\
\hline \multicolumn{2}{|l|}{ Total } & $1(100.00 \%)$ & $10(100.00 \%)$ & $5(100.00 \%)$ & $87(100.00 \%)$ & $2(100.00 \%)$ & $105(100 \%)$ \\
\hline \multirow{4}{*}{$\begin{array}{l}\text { There is significant stress in } \\
\text { parents of autistic children that } \\
\text { increases with increasing age of } \\
\text { children. They require attention } \\
\text { regarding their mental health. }\end{array}$} & Agree & $1(100.00 \%)$ & $7(70.00 \%)$ & $4(80.00 \%)$ & $53(60.90 \%)$ & $1(50.00 \%)$ & $66(62.90 \%)$ \\
\hline & Disagree & $0(0.00 \%)$ & $0(0.00 \%)$ & $0(0.00 \%)$ & $1(1.10 \%)$ & $0(0.00 \%)$ & $1(1.00 \%)$ \\
\hline & Neutral & $0(0.00 \%)$ & $1(10.00 \%)$ & $0(0.00 \%)$ & $9(10.30 \%)$ & $1(50.00 \%)$ & $11(10.50 \%)$ \\
\hline & Strongly agree & $0(0.00 \%)$ & $2(20.00 \%)$ & $1(20.00 \%)$ & $24(27.60 \%)$ & $0(0.00 \%)$ & $27(25.70 \%)$ \\
\hline \multicolumn{2}{|l|}{ Total } & $1(100.00 \%)$ & $10(100.00 \%)$ & $5(100.00 \%)$ & $87(100.00 \%)$ & $2(100.00 \%)$ & $105(100 \%)$ \\
\hline
\end{tabular}

\section{DISCUSSION}

It was alarming that by categorization of health-care medical processionals' according to the educational level, the Consultants who have completed their post-graduation medical education showed less awareness about autism, while the most junior House Officers who were fresh medical under-graduates or those doctors undergoing residency training like PG trainees were moderately aware in comparison. Other Medical Officers had an unsatisfactory level of awareness. However, there was significantly high knowledge amongst current medical students which can be an indicator to updated medical school curriculum or due to internet resources being more easily available. A Malaysian study also evaluated that senior medical students had significant knowledge regarding $A S D$ but were not clear about the medical student's source of knowledge, whether it is due to educational training or not ${ }^{11}$. Medical students will be the future doctors and as result in coming years, the professional knowledge gap might be curtailed as the diagnosis and treatment of this disorder will improve. Such a progressive step can pave the pathway to decreasing the number of cases of ASD and working towards an autism free society.

This study emphasizes that Pakistani physicians are not paying attention to ASD either due to lack of interest or limited awareness regarding its severity in the region. This is also highlighted by a study in Karachi which surveyed medical professionals regarding ASD, and the results revealed that more than half of the general practitioners have never heard of the term Autism ${ }^{12}$.

Another study conducted on medical students' level of ASD awareness showing level of knowledge in each year, compared that $4^{\text {th }}$ year students have a significant knowledge about diagnosis and symptoms of autism, more than final $5^{\text {th }}$ year students ${ }^{13}$. The researchers accentuate that the difference must be because of updating and recent revision of the medical curriculum ${ }^{14}$.

The responses varied per participant but regarding lack of knowledge about ASD's prevalence in the country; $41 \%$ were incorrectly unaware, reflecting upon how there is inadequate awareness about this disorder's increasing pattern in Pakistan. This requires immediate attention so that it can be timely addressed and resolved accordingly. The community must at least know that this disease exists in the Pakistani society. Many children are suffering because of insufficient diagnosis facilities and lack of information on the part of professionals.

A previous study conducted about the knowledge of autism; quantified that in healthcare there is low information about the symptoms of autism. The study reveals that only a minimum number of students examine the children who have autism during their clinical years in medical school ${ }^{15}$. The author concludes that there needs to be more 
exposure to the patients of autism for the students during their ward/clerkship duties ${ }^{15}$. This is necessary because clinical interaction is an efficient learning tool for practical clinical aspects involving inter-personal skills development.

Similar to this study, a huge number of medical students were surveyed in Karachi, which demarcated ASD as the swaying progressive disorder which disturbs the social communication and behavioural development of children. The aggregate score was low and emulate that there is a short-coming in information, edification, and cognizance about autism ${ }^{16}$. There is a need to include ASD in the medical and allied health sciences curriculum to improve the knowledge of professionals by continuous lectures. Professionals cannot counsel the parents satisfactorily when they themselves do not have sufficient knowledge about dealing with such children. Not only is there a need for specialists but also general physicians have to be made more aware.

The establishment of caregiver support groups is required for the affected children and their families ${ }^{10-17}$. The level of knowledge and understanding of the disease can have a significant impact on the treatment and families of deceased or affected children. Still, the country's policymakers are not giving pertinence to this matter as it deserves. The results highlighted show that the parents of Autistic children face a tough time while handling them because they show altered behaviour. This situation can cause stress to the parents, and over time, their mental health can be compromised. Researchers have confirmed that Autistic children are difficult to handle as they exhibit behaviours like irritability, aggressiveness, impulsivity, and hyperactivity due to which mothers are under great stress in comparison to fathers ${ }^{18}$. Age is also an important factor that contributes to the mental condition of the parents; for instance, in the early childhood Autistic children, the stress level is low in comparison to the middle and higher age ${ }^{19}$. Moreover, it is expected that with time, the mental and health issues of parents will increase with growth and development of the society.

Authorities must take this disorder seriously because special children need extra care, which can cause significant stress on the parents due to which the family relationships also get disturbed. Various studies have reported that when mothers deal with children with disabilities, there are higher symptoms of depression and increased psychological distress ${ }^{20}$.

To save mothers from this problem, it is necessary to take essential measures because, in families, the mothers' play a primary role in family relationship building. If the mothers have a poor mental health, then it may also have a bad impact on the growth and development of other children.

Autism is associated with being a constrained communication and monotonous disorder, alongside discrepancy in societal interaction. Most of the respondents believe that this is a brain disorder and not a mental illness.

In another study, the issue regarding autism awareness was analysed. The researchers examined the level of consciousness about autism and the stigmatization of individuals with ASD. The results showed that there is an absence of awareness among social circles regarding the autism disorder and the women are categorized by less slurring people as compared to men ${ }^{21}$.

When efforts for creating adequate awareness at all tiers of a society are worked upon, then due to the improved understanding, the aetiologies of a disorder can be minimized. Social-media and television campaigning, awareness walks, training workshops and other programs should be arranged. The involvement of medicine practitioners and medical students in these programs not only becomes an opportunity to refresh their knowledge but also becomes a medium for them to interact with the general public. Their visibility in them, makes it more authentic and serves as an encouragement for others to take the initiative of learning towards such medico-social matters. Such programs should also include the parents whose children are diagnosed with ASD so that they can be educated and taught how to deal with such type of children. Proper documentation of the diagnosis and treatment procedure should also be maintained and then shared with medical students and interns, so that they acquire experience regarding the process of the disorder's management.

It is expected that once there is increases awareness increases, a focus of direction will develop regarding ASD amongst relevant authoritative bodies. Researchers, specialists, medical students and other relevant personnel can work towards developing new methods and treatment plans by running research trial projects. The investigative and evaluative research progress will help understand the disease prevalence. This will help identify etiological agents alongside redesign and pave the path to speedy treatment modalities.

Limitations: The sample size of this study is not large and the respondent's affiliation to a centre is vague. Therefore, it cannot be categorized as multi-central, which would have given a broad representation. The questions accessed in the survey, are only focused upon child autism, while this disorder can also present in adults. The medical records and other specific documents of autism associated patients were not reviewed. Further, the survey did not ask about the details of features of an autistic person. Hence, the assessment of the knowledge of clinical symptomology is inadequate in this study.

\section{CONCLUSION}

Pakistan is a developing country with increasing levels of ASD with every passing year. This study reveals that social attentiveness regarding autism at all levels is insufficient, especially in healthcare professionals. It is crucial to increase awareness among medical professionals regarding this disorder so that the diagnosis and prognosis can be improved. Awareness regrading autism among medical students is satisfactory while knowledge amongst medical practitioners was unsatisfactory. There is a dire need for reform. Different seminars, advertisements, and social media campaigns are essential tools to be used for well defining the severity of this disorder to the community. More research investigative studies must be conducted on ASD, so that prevalence, aetiologies and treatment efficacies are recorded. Revision of medical curriculum for students and skills training is essential for doctors. 
Eventually, these efforts will not only be curbing the disorder's prevalence by beneficial treatment methods but also strategize preventive measures.

Disclosure: None

Conflict of interest: None

Funding disclosure: None to declare

Author contributions: NN: intellectual genesis, data analysis and manuscript writing, MT: Data analysis and Manuscript writing, SAA: Data analysis and Manuscript review, MM: Data presentation and Manuscript writing, SSAS: Data manipulation and Manuscript editing, SM: Data manipulation and Data cleaning, FF: Data manipulation and Data cleaning

\section{REFERENCES}

1. Nadeem T, Aftab R, Asad N. An overview of autism: is it treatable in Pakistan?. Pakistan Journal of Neurological Sciences (PJNS). 2019;14(3):4-5.

2. Reichow B. Overview of Meta-Analyses on Early Intensive Behavioural Intervention for Young Children with Autism Spectrum Disorders. Journal of Autism and Developmental Disorders. 2011;42(4):512-520.

3. Pavan E, Siniscalco D. Autism or New Autisms? A Psychologist Point of View. Autism-Open Access. 2016;6(4).

4. Imran N, Chaudry MR, Azeem MW, Bhatti MR, Choudhary ZI, Cheema MA. A survey of Autism knowledge and attitudes among the healthcare professionals in Lahore, Pakistan. BMC pediatrics. 2011 Dec 1;11(1):107.

5. Imran N, Azeem MW. Autism spectrum disorders: perspective from Pakistan. Comprehensive Guide to Autism. New York: Springer. 2014:2483-96.

6. Rafaq F, Haider SI, Latif S, ljaz S. The association between psychological capital, mental health, and burnout among specialists working in autism centers in Pakistan. J Environ Occup Sci. 2020;10(2).

7. Khan S, Qayyum R, lqbal J. Prevalence of Autism Spectrum Disorders (ASD) and Attention Deficit Hyperactivity Disorders (ADHD) among adult psych. Pakistan Armed Forces Medical Journal. 2019 Apr 19;69(2):419-23.

8. Azeem MW, Imran N. Pakistan and Autism. Encyclopedia of Autism Spectrum Disorders [Internet]. New York: Springer. 2016.

9. Hossain MD, Ahmed HU, Uddin MJ, Chowdhury WA, Iqbal MS, Kabir RI, Chowdhury IA, Aftab A, Datta PG, Rabbani G, Hossain SW. Autism Spectrum disorders (ASD) in South Asia: a systematic review. BMC psychiatry. 2017 Dec;17(1):1-7.
10. Boujarwah FA, Rozga A, Abowd GD, Arriaga RI, Oberleitner R, Pharkute S. Towards in-home collection of behavior specimens: Within the cultural context of autism in Pakistan. In2012 6th International Conference on Pervasive Computing Technologies for Healthcare (PervasiveHealth) and Workshops 2012 May 21 (pp. 9-16). IEEE.

11. Low HM, Zailan F. Medical students' perceptions, awareness, societal attitudes and knowledge of autism spectrum disorder: an exploratory study in Malaysia. International Journal of Developmental Disabilities. 2018 Mar 15;64(2):86-95.

12. Akhter M, Ashraf M, Ali A, Rizwan I, Rehman R. Integration of therapies in autistic children; a survey based in Karachi, Pakistan. JPMA. The Journal of the Pakistan Medical Association. 2018;68(10):1508.

13. Bakare MO, Tunde-Ayinmode MF, Adewuya AO, Bello-Mojeed MA, Sale S, James BO, Yunusa MA, Obindo JT, Igwe MN, Odinka PC, Okafor CJ. Recognition of Autism Spectrum Disorder (ASD) symptoms and knowledge about some other aspects of ASD among final year medical students in Nigeria, Sub-Saharan Africa. BMC research notes. 2015 Dec 1;8(1):454.

14. Khan A, Ferrari M. Wisdom of Young Adults with High Functioning Autism in Canada and Pakistan: A Cross-cultural Study. Journal of Education and Social Sciences. 2018;6(1):3.

15. Lord C, Elsabbagh M, Baird G, Veenstra-Vanderweele J. Autism spectrum disorder. The Lancet. 2018 Aug 11;392(10146):508-20.

16. Shaukat $F$, Fatima $A$, Zehra $N$, Hussein MA, Ismail $O$. Assessment of knowledge about childhood autism among medical students from private and public universities in Karachi. JPMA. The Journal of the Pakistan Medical Association. 2014 Nov $1 ; 64(11): 1331-4$.

17. Minhas A, Vajaratkar V, Divan G, Hamdani SU, Leadbitter K Taylor C, Aldred C, Tariq A, Tariq M, Cardoza P, Green J. Parents' perspectives on care of children with autistic spectrum disorder in South Asia-Views from Pakistan and India. International Review of Psychiatry. 2015 May 4;27(3):247-56.

18. Sabih F, Sajid WB. There is significant stress among parents having children with autism. Journal Rawalpindi Medical. 2008;33(2):214-6.

19. Anwar M, Tahir M, Nusrat K, Khan M. Knowledge, Awareness, and Perceptions Regarding Autism Among Parents in Karachi, Pakistan. Cureus. 2018;

20. Hassan K, Inam A. Factors contributing to stress among parents of children with autism. NURTURE: Journal of Pakistan Home Economics Association. 2013 Jan 1;7.

21. McCormack G, Dillon AC, Healy O, Walsh C, Lydon S. Primary Care Physicians' Knowledge of Autism and Evidence-Based Interventions for Autism: A Systematic Review. Review Journal of Autism and Developmental Disorders. 2019 Dec 4:1-6. 